\title{
Design and Evaluation of Player Experience of a Location-Based Mobile Game
}

\author{
Tara Carrigy, Katsiaryna Naliuka, Natasa Paterson, Mads Haahr \\ Trinity College Dublin \\ College Green, Dublin 2, Ireland \\ \{carrigyt, naliukak, patersn, mads.haahr\}@tcd.ie
}

\begin{abstract}
This paper reports on the design and evaluation of player experience of a Location-Based Mobile Game set in Dublin, Ireland in which players act as paranormal investigators hunting for ghosts and gathering evidence of paranormal activity. The paper focuses on players' experience of engagement and immersion, which was evaluated through a qualitative user study undertaken over a three-day period with the participation of 19 subjects. We first discuss the concept of immersion in gaming and then review related work before presenting the design and implementation of our prototype and the results of our user study. The results show that the experience succeeds in creating a high level of immersion at several stages in the game and that this immersion can be influenced by several factors including usability, control, modes of interaction, aesthetics, flow and, perhaps most significantly, choice of location.
\end{abstract}

\section{Author Keywords}

Engagement, Immersion, Location-Based Mobile Gaming

\section{ACM Classification Keywords}

H5.m. Information interfaces and presentation: User Interfaces-User-centred design; Evaluation

\section{INTRODUCTION}

Evaluation of players' experience in any form of digitally mediated gaming almost always refers to engagement, and specifically immersion, as desirable aspects of gameplay. In the general context of gaming, the term immersion is used to loosely describe the degree to which the player becomes involved or engaged in the game. Until recently understanding of immersion in computer gaming has mainly been developed in relation to $\mathrm{PC}$ and Console Gaming. As the new contexts of Pervasive Gaming,

Permission to make digital or hard copies of all or part of this work for personal or classroom use is granted without fee provided that copies are not made or distributed for profit or commercial advantage and that copies bear this notice and the full citation on the first page. To copy otherwise, or republish, to post on servers or to redistribute to lists, requires prior specific permission and/or a fee.

NordiCHI 2010, October 16-20, 2010, Reykjavik, Iceland.

Copyright 2010 ACM ISBN: 978-1-60558-934-3...\$5.00.
Augmented Reality (AR) Games and Location-Based Mobile Games (LBMGs) continue to evolve, game designers creating immersive gaming experiences will face unique and unprecedented challenges.

In this paper, we report on a study of player experience of a working prototype of a LBMG. Our primary goal was to test our assumptions about factors that contribute to the creation of engaging, compelling and immersive gameplay. Another motivation was to expand our understanding of players' engagement and to develop strategies for future development of this game. We begin by exploring established notions of engagement and immersion in gaming. Next we survey related work, focusing on research projects that evaluate player engagement, most specifically in the context of LBMG and more broadly in the context of Pervasive and AR Gaming. We then describe the design and implementation of the prototype and present our set-up and methodology for the qualitative evaluation study. Finally, we present the results of our study and discuss our findings with some strategies for further development.

\section{ENGAGEMENT AND IMMERSION}

In the general context of gaming, gamers, developers and researchers describe the players' experience of engagement or involvement in the game as immersion [3]. The term immersion is often used to describe the experience of losing track of the outside world and the boundaries of the magic circle, the imaginative space in which the game is played [1]. As we have already alluded to, initial understanding of the term immersion in gaming referred to the context of video and computer gaming and is therefore inevitably associated with VR technologies where the player enters or plunges into the virtual game world $[5,19]$. By stating that immersion is 'the sensation of being surrounded by a completely other reality' and 'the experience of being transported to an elaborate simulated place', Janet Murray's definition of immersion reflects this association [19]. In the VR and Virtual Environments (VE) research community immersion is commonly accepted as the qualities of a media that create sensory impact by surrounding the user $[9,29]$ and for decades this community has defined a set of 'immersive factors' almost exclusively in relation to the technology. For example, Slater proposes that 'immersion can be objectively assessed as the characteristics of a 
technology, and has dimensions such as the extent to which a display system can deliver an inclusive, extensive, surrounding, and vivid illusion of virtual environment to a participant' [29]. In contrast, however, Witmer suggests that immersion is a psychological state that can be influenced by the immersive tendencies of the individual participant [34]. In VE research, immersion is often measured by presence, 'the subjective experience of being in one place or environment, even when one is physically situated in another' [34]. In this context it is common to assume that presence can only be experienced through immersion in the VE $[29,34]$ and that greater presence leads to greater engagement.

Game theorists Salen and Zimmermann offer another perspective on immersion and presence when they observe that equating the experience of immersion with being 'sensually' transported to another separate, simulated reality so convincing that 'the player truly believes that he or she is part of an imaginary world' [27] is a fallacy that assumes the technologically mediated experience can become so sophisticated that it will be capable of 'fully illusionistic experiences indistinguishable from the real world' [27]. They propose that, in the context of gaming, the immersive fallacy of engagement through simulation is a one-dimensional approach to player engagement that 'misrepresents how play functions'. In contrast Salen and Zimmermann suggest that engagement 'occurs through play itself' in a process of double consciousness [27], whereby the players become engrossed in the game while simultaneously aware of the medium and the 'artificiality of the play situation' and thus perceiving play as something separate from, but nevertheless connected to the real world. This understanding of immersion does not disregard the power of simulation and the potential for engagement as a result of presence but it does present player engagement as a more complex and active process and this approach is especially relevant in the context of LBMG where the player is constantly shifting focus between the media and the physical environment and where gameplay moves fluidly between mediated and directly felt experience.

Player engagement and immersion in games is essentially performative and participatory and occurs as a direct result of active involvement, attention and interaction. In games, players are actively rather than passively engaged in the gameplay experience and therefore the quality of the players' interaction with the game system, through the game mechanics, is a key factor influencing immersion. Players enter the game through the game controls [3] and mastery of the game mechanics (the interface between the player and the game world) is the 'price of admission' [25] to the gameplay; as players learn how to use the game controls to play the game, their focus switches from conscious attention to internalised or tacit knowledge. This state of deep involvement shortens the distance between player and environment and results in immersion [4].
Players expect to be rewarded for the time, effort and attention spent learning, what Lindley refers to as, the 'gameplay gestalt' [17], the pattern of player interaction with the game system. Cairns and Brown's grounded theory of immersion suggests that as players pass through the barriers associated with the preliminary stages of psychological engagement, they are rewarded by a more imaginative experience that Cairns and Brown describe as total immersion [3,28]. Engagement and immersion therefore are part of a continuum of player experience, where initial engagement is associated with learning the game mechanics and is a prerequisite for deeper total immersion, which is the performance of the game mechanics in order to experience the gameplay. In this sense immersion is similar to flow, "the state in which individuals are so involved in an activity that nothing else seems to matter' [6] and where attention becomes so focused that 'sense of time is altered and sense of self is lost' [3]. Flow is the 'state at the boundary between engagement and immersion, of being totally absorbed in meeting the constantly unfolding challenge', which Lindley also refers to as immersion in performance [17]. For the purposes of this paper, we take the essential difference between experiences at either end of this continuum as follows: engagement is more active than immersion, and total immersion is closer to flow, the immersive state achieved when this activity is balanced by the players' skills and becomes so transparent that the player can experience deep but effortless involvement in the game $[3,25]$.

Immersion in gaming is considered to be more than the objective characteristics of the technology but also the subjective experience of the player as s/he plays the game. Recognising the different levels and stages of engagement and immersion in gaming, many researchers have emphasised that the experience of immersion is a transient and often fleeting state $[3,10,23]$. Part of the focus of this paper is to establish the factors that influence the players' experience of engagement and immersion as they move through the game, and in particular highlight those specific to the context of LBMG.

\section{RELATED WORK}

Researchers evaluating player experience in LBMGs have explored several factors that impact on player engagement and immersion, the most common focus being on the significance of location. Some LBMG blur the boundary between fact and fiction and leverage a combination of local legend and history to engage players, heighten their awareness and change their perception of the everyday places, where the games are located [2,22,34]. By evaluating REXplorer, a LBMG set in the Medieval city of Regensburg, researchers found that interweaving real world landmarks into the game's narrative, which they based on a mixture of local folklore and medieval history, added an authenticity to the gameplay that increased players' immersion [2]. Similarly, Visby Under and Frequency 
1550, are both LBMGs that use the immersive qualities of location to bring history to life and enhance player engagement [30]. Likewise Nisi et al, in their evaluation of Media Portrait of the Liberties, also report achieving deeper story immersion and an enhanced sense of place by overlapping and connecting narrative with location [20]. Riot! 1831, a locative interactive play, used audio to recreate the riots of 1831 and GPS technology to situate this media at the exact location in Bristol where the riots occurred [24]. Participants reported empathy with the people involved in the riots and a sense of "walking in their footsteps'. Research revealed a high level of immersion as direct result of mapping historic narrative onto relevant locations but also exposed that immersion is a transient state that can be affected by environmental factors associated with that location. Evaluation of LBMGs created using the Mediascapes toolkit highlights how making use of aspects in the physical environment can make the games more engaging. In recognising the difficulty but nevertheless high potential for immersion associated with a tight coupling of game content and environment, the Mediascapes researchers propose a strategy for 'designing for coincidence' whereby environment features or the behaviour of other people become aligned with the content of the game and are perceived by the player as highly engaging 'magic moments' [24]. Finally in relation to choice of location, researchers evaluating LOCUNET found that locating LBMGs in public spaces could have a negative impact on immersion if players felt inhibited or threatened by the presence of passers-by [7].

As well as transforming the players' perception of space with story, some researchers in the field of LBMG have explored techniques for augmenting space with visuals and sounds, which players will perceive as part of the hybrid gameworld, and have evaluated how this impacts on player engagement and immersion. For example, researchers compared player engagement in AR Façade to the previous PC version of the interactive narrative game Façade, and found that while the players of the AR version felt an increased sense of presence this did not necessarily result in increased player engagement [9]. Early user evaluation of TimeWarp, an outdoor augmented reality game played in the city of Cologne using a HMD, revealed that GPS tracking issues combined with the graphical realism of the AR objects, limited players' sense of presence because the objects floated around too much or did not appear to be real [28]. Evaluation of Interference, a LBMG using AR technology to superimpose 3D models on the mobile's live video stream, revealed that the aesthetics, which helped to blend the physical world into the game world, were the most significant factor contributing to player immersion in the game [33]. In relation to acoustic augmentation, research in VE has shown that accurately synthesized spatialised sound can increase presence [11] and researchers have shown how these techniques can be used to create realistic localised sound and a 3D audio soundscape that can be easily navigated by players and thus may increase immersion in Locative Games [5]. In Songs of the North, researchers used an audio interface to create an engaging location-aware mobile game because they felt that audio was a more appropriate medium for creating an immersive hybrid gameworld [15]. Related work has also shown that the mode of interaction in LBMG can affect player engagement. For example, REXplorer [2] uses gestural based interaction to physically engage players, while the iPerg [32] researchers recommend that the use of authentic or real world interaction models, such as walking around to discover elements of the game, can increase player immersion.

In addition to evaluations of LBMGs that focus on immersion and engagement, other related work includes research that has focused on methodology and models for evaluating player experience in the context of Pervasive Gaming. In the Pervasive GameFlow (PGF) model $[13,14]$, Jegers develops a model of player enjoyment of Pervasive Gaming based on the GameFlow model originally developed by Sweetser and Wyeth [31] and following Csikszentmihalyi's concept of flow as described in the previous section. With regard to immersion the PGF model recommends that pervasive games should enable players to shift focus from virtual and physical parts of the game and seamlessly transition between the gameworld and everyday contexts [13]. Ermi and Mäyrä's SCI model of immersion [10] is also useful for evaluation in the context of LBMG because it opens up the possibility of measuring player immersion from the perspective of the player. The SCI model of immersion considers player immersion from three perspectives: Sensory, Challenge-Based and Imaginative and in doing so encompasses key elements of gaming and is closely aligned to game models proposed by the gaming and game theory community, for example Lindley's Simulation, Gameplay and Narrative taxonomy [16,17].

\section{DESIGN AND IMPLEMENTATION}

Viking Ghost Hunt (VGH) is a location-aware adventure game, based on a Gothic ghost story set in Viking Dublin (800-1169). In this game the player assumes the role of a paranormal investigator and moves around the city hunting for ghosts, collecting evidence and solving the mysteries of haunted Viking Dublin. The game is designed as a singleplayer, immersive gameplay experience, in which the player is an active character in an unfolding drama. In order to progress the game, the player must unlock a sequence of location-specific narrative fragments, by completing a series of challenges and missions. Throughout the $V G H$ game design, we focused on two design goals: to give meaning to play by maintaining the aesthetics of role-play and to exploit the characteristics of the locations by integrating them into the game to create an engaging gameworld. 


\section{Role-Play}

In order to preserve the illusion or magic circle the players' assumed role as a paranormal investigator must persist at all times. We set this up by presenting all of the aspects of gameplay, including the aesthetics, challenges and modes of interaction, in the context of paranormal investigation. Equipped with a paranormal investigation device and an audio headset, the players' goal is to conduct investigations by hunting down ghosts and gathering evidence, by making audio recordings or taking photographs of ghostly apparitions. Players use Google maps to search for the location of an alleged haunting in Viking Dublin. Players begin their investigation by using the Radar (see Figure 1) to locate paranormal activity in the region. This Radar also includes an Electromagnetic Frequency (EMF) meter, which informs players of the levels of paranormal activity and the strength of the manifestations. A dowsing meter (based on the metaphor of dowsing rods used to divine water or paranormal activity) helps to guide the player in the direction of the haunting. Once the player has located the paranormal activity, s/he can begin to collect evidence that will substantiate the alleged haunting. Players use the Frequency Scanner (see Figure 1) to tune in and decode the static, in order to reveal the ghostly voices in the white noise, in a process reminiscent of Electronic Voice Phenomenon. Ghost View can be used to photograph visual manifestations. Players' can review their progress in Casebook, a database that stores players' evidence and contains extra narrative about the ghosts.

\section{User Interface Design}

The User Interface (UI) design was guided by the aesthetics of role-play, as described above, and the intention of facilitating embodied interaction [8] with the game as is appropriate for situated and location-based gaming. To these ends, we designed the UI to look and feel like a tool for paranormal investigation and to evoke a similar cognitive process as someone investigating a radioactive area with a Geiger counter. By employing a visual style of presentation reminiscent of mechanical devices of the past, we established a set of affordances that suggested the appropriate mode of interaction to the user while simultaneously reinforcing the aesthetics of role-play and atmosphere of the game. By way of engaging the player in the processes of searching for and collecting evidence, we applied the principles of direct manipulation by enabling phenomenological interaction via touch screen gestures and multimodal feedback in response to players' actions and movements. As much as possible, the intention was to reference real world actions as interaction metaphors so that players' could easily master the gameplay gestalt by leveraging their existing tacit knowledge [4,17]. For example, navigating the gameworld is achieved through the activity of searching for ghosts by walking around the location and using the Radar to detect paranormal activity. The players' proximity to the ghost is indicated using a combination of visual representation and a fluctuation in audio and tactile feedback, hence the players can navigate the gameworld using a combination of sound, sight and touch. Collecting evidence by photographing ghosts using the camera function is also an intuitive and familiar mode of interaction.
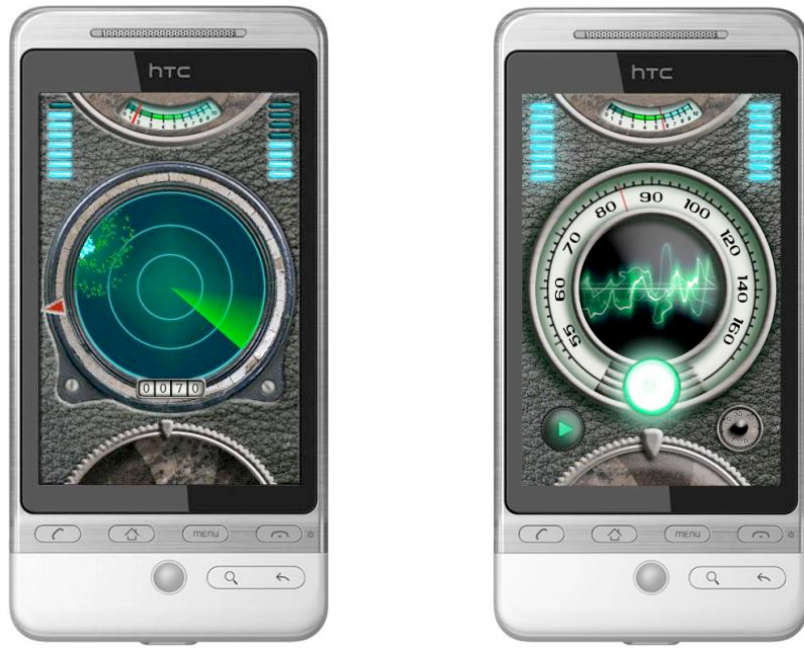

Figure 1. Radar (left) and Frequency Scanner (right).

\section{Use of Location}

We set the game around St. Audeon's, a Medieval church and public parkland situated within the Old Viking City Walls of Dublin because of its historical and thematic relevance and potential for a varied player experience. With the intention of blurring the distinction between fact and fiction, we based the game's narrative on a mixture of local ghost stories and urban myths as well as factual history. We included contrasting locations in the mission design in order to test the impact of location on gameplay. The main location was ideal for the theme and atmosphere of the game and we were able to integrate features such as the Medieval style church, windy ramparts, high stone walls, dark archways and damp and eerie laneways; all classic motifs of the Gothic ghost story genre. Narrative was used to draw player's attention to physical features in the environment and integrate them as part of the gameworld. Dialogue, in combination with sound effects, helped players imagine a scene from the past. For example, in the Viking era, the River Liffey was much broader than it is today and hence the grassy area outside the old city walls was once a beach; we recreated this scene using sound effects and descriptive narrative. Similarly, the narrow laneway and medieval staircase at the side of the church was transformed into the 'Lane of Hell' by a dense interplay of atmospheric dialogue enhanced by realistic sound effects. Exploiting the characteristics of the locations is depends on presenting relevant content to the player in relation to their position in the gameworld. We set this up by specifying regions where the assets would be triggered and then used GPS to detect when players had entered these regions. Since GPS technology is prone to lagging and errors, efforts were made to minimize inaccuracies by creating large regions 
and positioning them at locations with good GPS reception (e.g. open areas not shadowed by trees and buildings). In situations where we needed to place content close to buildings, for example in the 'Lane of Hell' scenario, we used the physical properties of the space to compensate for the lack of GPS updates in this region. In this example, the assets were triggered by regions at either end of the lane thus limiting the experience of this content to the confines of the lane. Despite this approach, the resolution remained fairly low in precision and therefore the assets could only be loosely mapped to specific physical features.

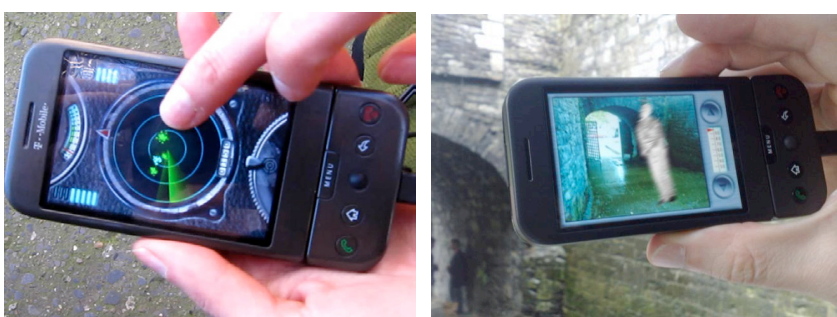

Figure 2. Using Radar's touch screen zoom (left) and Ghost View (right).

\section{Augmented Reality}

Various Augmented Reality (AR) techniques were used to transform and augment the players' visual and auditory perceptions of the location. With the aim of creating the illusion that the ghost is actually present in the player's surroundings, the players' view of the scene is augmented by animations displayed on top of the live video stream in the viewfinder of the camera. The effect is perceived as more realistic when the ghost is anchored to a point in the scene and therefore appears to remain stationary in the physical surroundings even when the player moves the camera. Due to the impracticalities of placing optical markers into a public space and the expensive overhead of performing real-time image analysis, it was not possible to employ computer vision techniques to anchor the ghost overlay within the scene. Instead we tracked the direction of the viewfinder using the device's orientation sensors and although (due to the unreliable nature of the sensor data) the ghost representation was not completely fixed within the scene, we speculated that the visual floating effect would be credible in the context of ghostly apparitions. Acoustic augmentation of the location was achieved by using spatialisation and reverberation techniques [19] to realistically match the game's sound effects to those naturally occurring in the environment and as a way of deliberately creating ambiguity between the game and the real world. In addition a localised soundscape was created by varying the volume of the sound effects in response to the players' movements around the hybrid gameworld. Audio and visual augmentations were constrained by the resource limitations of the Android development platform which requires the assets to be loaded into memory on demand. The implication of this constraint is that the file size needed to be very small thus hampering the creation of realistic effects and also the assets had to be positioned far enough apart to give the device time to re-load each of the individual assets as required.

\section{EVALUATION}

We evaluated the VGH game by running a series of field trials over a three-day period. The 19 participants who took part had varying degrees of prior experience of gaming and ranged in age from 18-48. We evaluated the participants' response to the game using a post-game qualitative questionnaire loosely based on a combination of established models of player engagement and immersion [10,14,31,34]. The questionnaire included both open ended and bounded questions designed to obtain feedback on engagement, control, usability, presence and impact of location. While an in-game evaluation technique such as 'think-aloud' may have captured a more immediate and visceral response to the game, this was not practical due to resource limitations. We also captured data during the game using the system logs. While this data did support the information gathered by the retrospective questionnaires it did not offer anymore insight into the player experience and therefore we will not present this data as part of our evaluation.

\section{RESULTS AND OBSERVATIONS}

\section{General Overview}

Evaluation of the user study results reveals that overall participants were engaged in the game. Feedback from three of the bounded questions illustrate that the majority of participants (79\%), agreed or strongly agreed, that it was a fun experience, time passed quickly and they felt engaged by the game. All participants reported varying levels of engagement at different stages of the game. In general, the findings support previous research that indicates immersion is a variable and transient state, which progressively deepens as players became more skillfully engaged in the gameplay $[3,4,17,23]$. Many players describe becoming more immersed as the game progressed and as they became more comfortable using the UI or accomplishing the challenges and as the story began to unfold.

"It was more immersive once I got used to the UI and the story started to link one sighting to the next"

"I didn't feel immersed until near the end, the beginning isn't so engaging, as I was trying to get used to how to control the game and find the evidence at the same time"

\section{Interaction and immersion in gameplay}

The user study findings show that by far the most engaging aspects of gameplay were connected to the mastery of the game mechanics. For the most part, players found the search mechanic satisfying and enjoyed the process of physically moving around the environment hunting for evidence of paranormal activity and the sense of achievement when they accomplished the challenge.

"The gameplay was lovely, the different modes and the physical moving around to track things down." 
Overall players used the multimodal features of the Radar interface as a guide when searching for ghosts; 79\% of participants mainly used the graphical interface but also used audio and tactile feedback to support the visual feedback. Since information about the position of the ghost was presented in different ways, players could choose their preferred method or tools to help them find the ghosts. For example, testers might focus on visual, tactile or audio feedback or a combination all features.

"I used the proximity meter (i.e. number readout) to determine my distance."

"The sound effects and the pulsing of the handset helped point me in the right direction."

Many players described collecting evidence as the most immersive part of the game because they were simultaneously engaged in the activity of recording evidence while also receiving a reward for progressing in the game in the form of a story fragment.

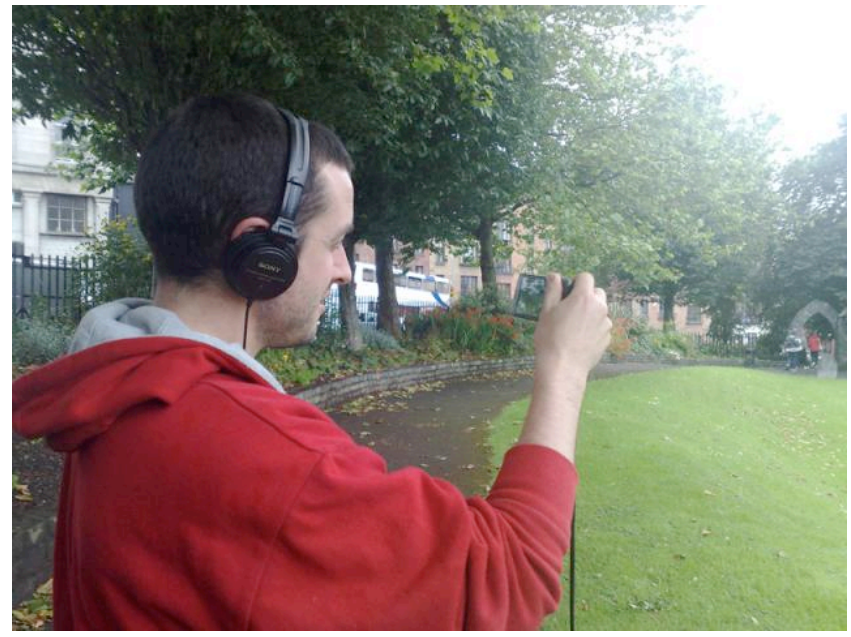

Figure 3. Player interacting with ghosts in St.Audeons.

"It felt satisfying. The ability to find audio and images was a good mix. I never got bored searching. The most immersive part was after I'd located a ghost or artifact, and am trying to record it"

"Felt most immersed with dialogue and with use of camera, felt engaged when I caught the floating apparitions. Dialogue gave more direction and hence more interactive."

\section{Impact of control}

The study shows that GPS and usability issues negatively impacted on the player experience with the result that only $63 \%$ of players said they felt in control of the game. In the main, efforts to reduce the negative impact of GPS errors were successful. In particular the strategy implemented in the 'Lane of Hell' region, as described in the design section, resulted in the delivery of relevant content at appropriate locations despite lack of GPS coverage. Nevertheless some players did experience bad GPS service and this caused a high degree of distraction and frustration.
"The most difficult part of the game was the device reliability: sometimes the GPS 'ghost' would point me in the wrong direction."

Usability issues occurred due to latency and unresponsiveness of the touch screen and sensors as a result of resource limitations while new assets were being loaded. For example sometimes the zoom function of the Radar became slow to react and therefore participants found it awkward to use and often "required moving slowly and stopping regularly for re-calibration"

\section{Impact of location}

A significant finding of the user study was the impact that the location had on player engagement and immersion. Almost all the participants agree $(84 \%)$ that the location contributed to the overall game experience because the historical significance of the main site gave context to the narrative, while the atmosphere of the place enhanced the players' perception of the supernatural theme of the game.

"The historical significance of the place influenced my perception of the game"

Most participants had not previously visited this site and commented that this added a sense of intrigue to the location.

"The church had walls, nooks and crannies; also, the alleyway by the side of the church, the high walls, the archway all added to a kind of 'mysterious' atmosphere."

Participants confirmed that their experience of the game varied in relation to the ambience of the locations; the aesthetics of the main site were much more conducive to the atmosphere of the game than the commercial streets and housing estates at the periphery of the historic site. The relative remoteness of the church grounds also enhanced player immersion because the location was quiet and less populated than the urban streets and therefore afforded uninterrupted engagement.

"The isolated lane around the side of the church was more atmospheric and hence I felt more immersed"
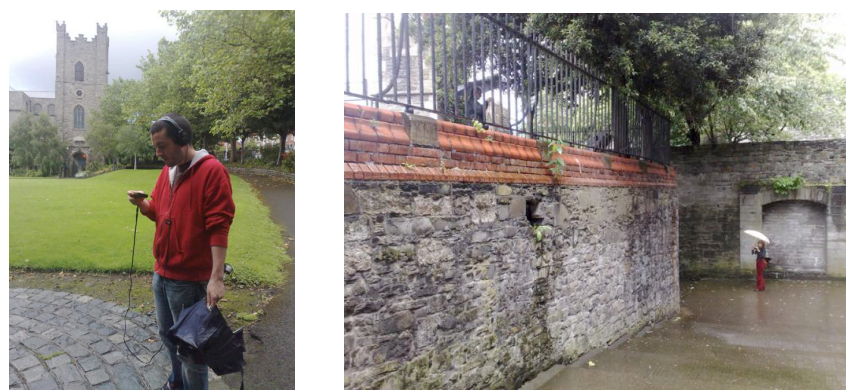

Figure 4. Players at St. Audeon's Arch and the 'Lane of Hell'.

Players' noted that the use of headphones helped to engage them in the game by reducing distractions without completely disconnecting them from the ambient sounds of 
the location, which were still hear audible with the headphones on.

"The only thing that distracted me was a passing ambulance as I caught a sound ghost".

Players found the locations on the busy urban streets outside of the church afforded a less immersion experience due to distractions such as noise levels, passersby and traffic safety issues. It was difficult to hear the audio at the points located near to the roadside due to the traffic noise and some felt that safety was an issue in this scenario. While a few players felt self-conscious playing the game in public, especially if they were in the way of other pedestrians when they stopped in the middle of the pathway to listen to the narrative, for the most part players took less notice of other people in areas such as the park as they felt these areas were already designated for play and one participant commented: "I was fully immersed and didn't take notice of tourists and workmen around me".

\section{Role-play and aesthetics}

In general, participants agreed (84\%) that the visual appearance and sounds of the UI made the device feel more like a tool for paranormal investigation than a phone. In comparison less participants $(68 \%)$ reported feeling like a paranormal investigator when they were playing the game and therefore we can conclude that while the audio and visual aesthetics supported the impression that the device was real, the game mechanics did not support role-play to the same extent. Nevertheless, participants described several features of the UI that they associated with paranormal investigation and felt that the headset helped make the role-play feel genuine.

"I did feel like a paranormal investigator, hunting things down was great and twiddling with the knobs to tune in"

"I had the device to find the object and I felt like the ghost hunters from the film"

In addition, and reflecting the cumulative nature of immersion, many participants reported feeling more engaged in the role-play as the game progressed.

"After a few discoveries it was easy to get into the game and to feel more like an investigator."

Overall the findings show that participants perceived the game assets, and in particular the audio assets, as realistic and this contributed to player engagement. Participants reacted emotively to the dialogue spoken by the audio ghosts and many described the experience of listening to these ghosts as 'scary', 'spooky', 'haunting', 'atmospheric', 'realistic', 'believable' and as a result 'engaging', 'immersive' and 'entertaining'.

"I found it [the ghost speaking] one of the most immersive features of the game - really enjoyed it."

Many players commented that when the ghosts spoke to them, the experience felt very personal and rewarding and this helped to engage the player in the game because it communicated the players' progress.

"Felt kind of like a personal experience - like it was tailormade for me and the ghost was egging me on to the next clue"

$79 \%$ participants felt the game audio sounded natural in the environment and some testers refer to incidences when the game sound blended with the ambient sounds of the environment in a manner that created ambiguity as to which was virtual and which was real.

"The natural sounds of the city were kind of a nice extra layer of atmosphere rather than a distraction, there were children playing and a church bell ringing and a little bit of wind."

Players also felt rewarded and satisfied when they encountered and photographed visual assets and their remarks reveal the cumulative success of this game mechanic.

"Increasing use of visual (rather than auditory) clues heightened the experience as the game unfolded."

Many participants reported feeling 'engaged', 'entertained', 'excited', 'surprised' or 'satisfied' when they encountered a visual manifestation of a ghost, others described the visual ghosts as 'scary', 'realistic', 'cool' and 'good fun' and one player remarked 'the effect of it floating there was good, especially the first time when I wasn't expecting it'. In general, the visual assets, were not as convincing as the audio ghosts. It seems that the limited animation sequence negatively impacted on the user experience and some participants' remarks reveal that the animations were barely perceptible.

"Didn't seem real. Could have made the ghost appear like it was more than just a picture and part of the scenery."

\section{Narrative Immersion}

The most diverse feedback we received was in relation to narrative; while some players remained highly engaged with the game mechanics and less involved by the content of the story, others found that performing these mechanics gave players access to immersion in the narrative and "when clues began, the game was more exciting"

"I wanted to figure out how to get the evidence... but I was not really interested in what the evidence was"

"Story interests me most. I just like the 'adventure' idea of tracking down the information."

Some players noted that gameplay was a more essential aspect to the game but had they had more time they would have explored the narrative elements in more depth, while others stated that they were not that interested in using the casebook because they were more interested in gameplay.

"The interaction with the game was more about achievement ....I would like to play the game again and 
focus more on the story and not be distracted figuring out the equipment."

Most players felt they did not have time to use the casebook to find out more about ghosts but some players remarked that if they were playing under normal circumstances they would have used casebook more. Many players admitted to skimming the game and only did what they had to do to get to the end.

"I skipped this bit [reading casebook] as I was lagging behind and felt I needed to move on to the next find."

The players who did use casebook reported that the backstories were interesting and increased their empathy with the characters. As with the other game features, immersion in the story increased as the game progressed.

"As the game progressed, I certainly did get more immersed. As you find the different clues and items and realise you are following a particular ghost character it becomes more engaging"

\section{DISCUSSION}

\section{Challenge-based Immersion}

The results of the study show that engagement in play itself and immersion in performance were experienced by the players as the most immersive aspects of the VGH game. Furthermore the study shows that engagement in the game mechanics is cumulative, not only because as players' skills increase they become absorbed in a state of flow, but also because by engaging in these mechanics, players become exposed to other gameplay elements such as reward and narrative. We found that players entered the game through the game mechanics and in this sense our study of immersion supports the research we reviewed at the beginning of this paper $[3,10,25,27]$. Reflecting Ermi and Mäyrä's evaluation of PC games, we found that when we applied their SCI model of immersion [27] in the context of LBMGs, challenge-based immersion was also the type of immersion most frequently experienced by the players. For many players, recording audio or photographing ghosts was the most immersive part of the game because as well as creating atmosphere and communicated elements of the narrative, collecting evidence helped the players advance in the game. The study also indicates that this form of immersion is more easily achieved in the context of LBMGs when challenges are presented to the player in the form of easily controlled real world interactions.

\section{Real World Interaction}

The results of the study confirm previous research indicating that in the context of LBMGs the most successful game mechanics are those that combine real world activities as part of the core interaction $[2,32]$. For example, the search mechanic involves players walking around hunting for ghosts and the game supports a phenomenological approach to this challenge by providing multimodal feedback to players' movements thus enabling players to focus on the feedback which they found most intuitive. Comparing user response to our two modes of collecting evidence (recording audio and taking photographs) also supports the argument that more familiar, 'everyday' interactions are easier to perform in this context because they are more immediately intuitive and serve as more expedient modes of interaction because they rely on players' existing tacit knowledge [4,17]. These findings compliment the evaluation of REXplorer where players faced difficulties performing gestural interactions due to their novelty and associated learning curve [2]. As with previous research [7] our study reveals that players were less likely to experience immersion when they felt conspicuous playing the game in a public space however we also found that players were less inhibited when performing more 'everyday' interactions in public spaces designated for leisure activities. This confirms Jegers recommendation that LBMG should support a 'seamless transition between the gameworld and everyday contexts' in a manner that does not break the social norms associated with these contexts [13].

\section{Control}

Since players enter the game through the game controls, usability issues can have a negative effect on immersion. At times GPS errors disrupted players' enjoyment of the game and this well documented issue remains for anyone designing a LBMG. However in our user study, despite the fact that many participants did not always feel fully in control of the game many reported high levels of engagement and immersion and this seems to confirm Norman's theory $[3,17]$ that if the overall experience is interesting enough then users will be prepared to put up with minor usability issues. While some participants experienced difficulties at the beginning of the game, the findings of the study show that as players progressed, for the most part control ceased to be an issue. Having said that our recommendation for UI design for a LBMG is that the interface be made as simple and easy to control as possible because players have less tolerance for learning new interactions in this scenario and the inevitable GPS issues already create a difficulty level that need not be further extended by a complicated interface. Our study confirms Jegers' recommendation that in relation to control, players in the context of LBMG need to be able to easily pick up the game and begin playing [13].

\section{Augmented Reality}

The results of the user study show that choice of location can impact dramatically on sensory immersion in the context of LBMGs and by enhancing and transforming the players' perception of their surroundings, atmosphere can become a unifying and highly engaging aspect of the game. The findings reveal that the aesthetics of the assets most effectively contribute to the players' sensory immersion when they become subtly blended with the real world; for example, when the ambient sounds of the game merged with the environmental sounds of the location. The results 
also reveal that if this juxtaposition is not smooth enough, as was the case with the AR visuals, then the players will perceive it as jarring and unconvincing and hence a disruptive rather than engaging factor. This supports the findings of the evaluation of TimeWarp [28] as reviewed in the related work section. While to some degree the floating effect of the AR visuals was perceived as acceptable within the ghostly theme and context of the VGH game, it is clear that the visuals need to be more integrated into the surroundings in order to achieve a credible visual effect. Stabilising the visual overlays within the scene would also enable designers to employ the cinematic technique of 'mise-en-scene' whereby explicit visual attention could be drawn to specific landmarks as the player views them through the lens of the device. This could also address the fact that some players felt the game did not facilitate enough engagement in the surrounding environment.

"Paradoxically the game encourages looking at the screen more than the surroundings."

These findings highlight one of the unique challenges of creating presence in the context of LBMGs using the processing power of a mobile phone. Given the limited technical resources of the device and the significant potential of leveraging the location, it seems that at the moment the greatest potential for creating presence and sensory immersion in this context lies in the power to subtly transform the existing characteristics of the location into a sensually engaging gameworld by enabling players to simultaneously focus on virtual and physical parts of the game.

\section{Imaginative Immersion}

Overall we found that players needed to achieve a degree of effortless engagement before they could fully experience imaginative immersion and therefore this type of immersion is not dissimilar to immersion in the general gaming context $[3,10]$. The choice of location can influence the level of imaginative immersion not only by supporting the atmosphere and narrative but by also ensuring safety and lack of distraction. In some instances the narrative and audio completely transformed the players perception of the landscape, for example, one participant referred to the 'wistful description of the River Liffey' and the soundscape that described the original course of the river.

"The church and surroundings were smashing, really wonderful moment standing outside it and being told about the shores of the Liffey once being underfoot."

We speculate that part of the reason it was easy for players to imagine this alternate scene was the fact that the location where the game was situated was not that dissimilar to the one being described and was conducive to imaginative immersion due to the aesthetics of the location and lack of distractions. These findings contrasts with the evaluation of Riot! 1831 where researchers found that lack of visible structures that related to the experience made it difficult for participants to achieve imaginative immersion to the extent that they almost had to close their eyes in order to block out their physical surroundings [23].

Our study reveals that while many aspects of the gameplay contributed to imaginative immersion, for example roleplay and the atmosphere of the location, the story itself was not a hugely engaging aspect of the game. While players enjoyed the activities associated with discovering the evidence and piecing together the clues in order to proceed to the next find, very few participants spent time exploring the stories behind the evidence, which together made up the over-arching narrative of the game. There are a number of reasons for this outcome, the first being that, as described above, players needed to master the game controls in order to gain access to the unfolding narrative. However the main reason was that this mode of interaction, reading text on a small screen, is inconvenient in an outdoor location-based context. In future developments of the VGH game we intend to focus on conveying all of the narrative through short audio and visual assets and in doing so take advantage of the more accessible descriptive potential of these mediums.

\section{CONCLUSION}

In this paper we have observed that player experiences are cumulative. As players learned to gain control of the game mechanics they achieved deeper immersion in the narrative and imaginative aspects of gameplay. Supporting engagement and immersion in LBMGs is also dependent on utilising appropriate real world interactions and carefully selecting locations in relation to thematic relevance, atmosphere, aesthetics, safety issues, lack of potential distractions and social context. By leveraging the existing characteristics of the location, Augmented Reality techniques can be used to transform the surroundings in order to create a hybrid gameworld where the player can become immersed in the flow of play while simultaneously remaining connected to the real world.

\section{ACKNOWLEDGMENTS}

We wish to thank the rest of the $V G H$ team (Roisin Cotton, Daniel Crowley, Alan Duggan, Tina Hedayet, Soren Kristian Jensen and Sean O'Reilly), the NDRC and all those who participated in the user study.

\section{REFERENCES}

1. Adams, E. and A. Rollings. Fundamentals of Game Design. Prentice Hall, Upper Saddle River, NJ, USA, 2006.

2. Ballagas, R., Kuntze, A. and Steffen P. W. Gaming Tourism: Lessons from Evaluating REXplorer, a Pervasive Game for Tourists. In Pervasive Computing 2008, LNCS vol 5013, Springer (2008), 244-261

3. Brown, E., \& Cairns, P. A grounded investigation of game immersion. In Proc. of CHI 2004, ACM Press (2004), 1279-1300. 
4. Calleja, G. Revising Immersion: A Conceptual Model for the Analysis of Digital Game Involvement In Situated Play. In Proc. of DiGRA Conference (2007).

5. Cater, K., Hull, R., Melamed, T., and Hutchings, R. An investigation into the use of spatialised sound in locative games. In Ext. Abstracts CHI 2007, ACM Press (2007), $2315-2320$

6. Csikszentmihalyi, M. Flow: The Psychology of Optimal Experience. New York: Harper and Row, 1990.

7. Diamantaki, K., Rizopoulos, C., Charitos, D \& Tsianos, D. "LOCUNET: Location-based games as media communicating social meaning". In Souza e Silva, A. \& Sutko, D. (eds.), Digital Cityscapes: Merging Digital and Urban Playspaces, Peter Lang Pub, 2009.

8. Dourish, P. Where the Action Is: The Foundations of Embodied Interaction. Cambridge: MIT Press, 2001.

9. Dow, S., Mehta, M., Harmon, E., Mateas, M., Presence and Engagement in an Interactive Drama. In Proc. of CHI 2007, ACM Press (2007).

10. Ermi, L. and Mäyrä, F. Fundamental Components of the Gameplay Experience: Analysing Immersion. In Proc. of the DiGRA, (2005) 15-27.

11.Hendrix, C., \& Barfield, W. The sense of presence within auditory virtual environments. Presence: Teleoperators and Virtual Environments, 5(3) (1996), 290-301.

12. Herbst, I., Braun, A. K., Mccall, R., Broll, W., Timewarp: interactive time travel with a mobile mixed reality game. In Proc. MobileHCI '08, ACM Press (2008), 235-244.

13. Jegers, K. Pervasive Game Flow: Understanding Player Enjoyment in Pervasive Gaming. Computers in Entertainment. ACM Press (2007).

14. Jegers, K. Pervasive GameFlow: Identifying and Exploring the Mechanisms of Player Enjoyment in Pervasive Games. Ph. D. Thesis, Dept of Informatics, Umeå University (2009).

15.Lankoski, P.; Heliö, S.; Nummela, J.; Lahti, J.; Mäyrä, F.; Ermi, L. A Case Study in Pervasive Game Design: The Songs of North. In Proc. of NordiCHI, ACM Press (2004), 413-416.

16. Lindley C. Game Taxonomies: A High Level Framework for Game Analysis and Design. Gamasutra (2003)

http://www.gamasutra.com/view/feature/2796/game_tax onomies_a_high_level_.php

17. Lindley, C. A. 2004. Ludic Engagement and Immersion as a Generic Paradigm for Human-Computer Interaction Design. In Proc. of ICEC2004, Springer (2004), 3- 13.

18. McMahon, A. "Immersion, Engagement and Presence: A Method for Analysing 3-D Video Games." In Wolf, M.J.P and Perron, B. (eds.), The Video Game Theory Reader. London: Routledge, 67-86, 2003.
19. Murray, J. H. Hamlet on the Holodeck: The Future of Narrative in Cyberspace. Free Press, New York, 1997.

20. Nisi, V., Oakley, I. and Haahr, M. Location-Aware Multimedia Stories: Bringing Together Real and Virtual Spaces. In Proc. of ArTech, ACM Press (2008).

21. Norman, D. A. The design of everyday things. New York: Doubleday, 1988.

22. Paterson, N., Naliuka, K., Jensen, S.K., Carrigy, T., Haahr, M., Conway, F. Design, Implementation and Evaluation of Audio for a Location Based Augmented Reality Game. In Proc. of AMC Fun and Games, ACM Press (2010).

23. Reid, J., Geelhoed, E., Hull, R., Cater, K., Clayton, B. Parallel Worlds: Immersion in location-based experiences. In Proc. of CHI 2005, ACM Press (2005).

24. Reid, J. Design for coincidence: incorporating realworld artifacts in location-based games. In Proc. of ACM DIMEA, ACM Press (2008).

25. Rigby, S., Ryan, R., The Player Experience of Need Satisfaction ftp://ftp.immersyve.com/PENS_Sept07.pdf

26. Rouse, R. Game Design: Theory and Practice. Wordware Publishing, Texas, 2005.

27. Salen, K. and Zimmerman, E. Rules of play: game design fundamentals. MIT Press, Cambridge, Massachusetts, 2004.

28. Seah, M. and Cairns, P. From Immersion to addiction in videogames. In Proc. of HCI 2008, vol 1 BCS, 55-63.

29. Slater, M. and Wilbur, S. A framework for immersive virtual environments (FIVE): speculations on the role of presence in virtual environments. Presence: Teleoperators \& Virtual Environments, Vol. 6 Issue 6, (1997) 603-617

30. Souza e Silva, A., and Sutko, D. M. Digital cityscapes: merging digital and urban playspaces. Peter Lang Publishing, 2009.

31. Sweetser, P. \& Wyeth, P. GameFlow: a model for evaluating player enjoyment in games. In $A C M$ Computers in Entertainment, 3(3) ACM Press (2005), 124.

32. Waern, A., Montola, M., Stenros, J. "The three-sixty illusion: designing for immersion in pervasive games". In Proc. of CHI 2009, ACM (2009)

33. Wetzel R., McCall R., Braun A-K., Broll W. Guidelines for Designing Augmented Reality Games. In Proc. of the 2008 Conference on Future Play, ACM Press (2008).

34. Witmer, B. G., \& Singer, M. J. (1998). Measuring presence in virtual environments: A presence questionnaire. Presence-Teleoperators and Virtual Environments, 7(3), 225-240. 